\title{
Rectified momentum transport for a kicked Bose-Einstein Condensate
}

\author{
Mark Sadgrove $^{1,2}$ * Munekazu Horikoshi ${ }^{1}$, Tetsuo Sekimura ${ }^{1}$, and Ken'ichi Nakagawa ${ }^{1,2}$ \\ ${ }^{1}$ Institue for Laser Science, The University of Electro Communications, Chofushi, Chofugaoka 1-5-1, Japan and \\ ${ }^{2}$ Japan Science and Technology Agency
}

(Dated: October 31, 2018)

\begin{abstract}
We report the experimental observation of rectified momentum transport for a Bose-Einstein Condensate kicked at the Talbot time (quantum resonance) by an optical standing wave. Atoms are initially prepared in a superposition of the 0 and $-2 \hbar k_{l}$ momentum states using an optical $\pi / 2$ pulse. By changing the relative phase of the superposed states, a momentum current in either direction along the standing wave may be produced. We offer an interpretation based on matter wave interference, showing that the observed effect is uniquely quantum.
\end{abstract}

The current interest in rectified atomic diffusion, or atomic ratchets, may be traced back to fundamental thermodynamical concerns [1] and also the desire to understand the so-called "Brownian motors" linked to directed diffusion on a molecular scale [2, 3]. Abstractly, the ratchet effect may be defined as the inducement of directed diffusion in a system subject to unbiased perturbations due to a broken spatio-temporal symmetry.

Given the scale on which such microscopic ratchets must work, it is not surprising that the concept of quantum ratchets has recently augmented this area of investigation. The addition of quantum effects such as tunneling gives rise to new ratchet phenomena such as current reversal [4]. Whilst early quantum ratchet investigations, both theoretical and experimental, have focussed on the role of dissipative fluctuations in driving a ratchet current [5], recent theory has considered the possibility of Hamiltonian ratchets, where the diffusion arises from Hamiltonian chaos rather than stochastic fluctuations [6]. This has lead to proposals [7, 8] and even an experimental realisation [9] for ratchet systems realised using atom optics, in the context of the atom optics kicked rotor [10] where periodic pulses from an optical standing wave kick atoms into different momentum states.

It is generally accepted that a ratchet effect cannot be produced without breaking the spatio-temporal symmetry of the kicked rotor system. In Ref. [9], a rocking sine wave potential was combined with broken time symmetry of the kicking pulses to effectively realise such a system in an experiment. Other schemes involve the use of quantum resonance (QR) to drive the ratchet effect. At QR, atoms typically exhibit linear momentum growth symmetrical about the initial mean momentum. However it has been suggested that merely breaking the spatial symmetry of the kicked rotor at QR may be sufficient to produce a ratchet current [1]. In this letter we present the first experimental evidence of such a resonant ratchet effect in which the underlying mechanism is purely quantum. Our system uses a Bose-Einstein condensate (BEC) kicked by an optical standing wave [12], but there is no asymmetry in either the kicking potential

*Electronic address: mark@ils.uec.ac.jp

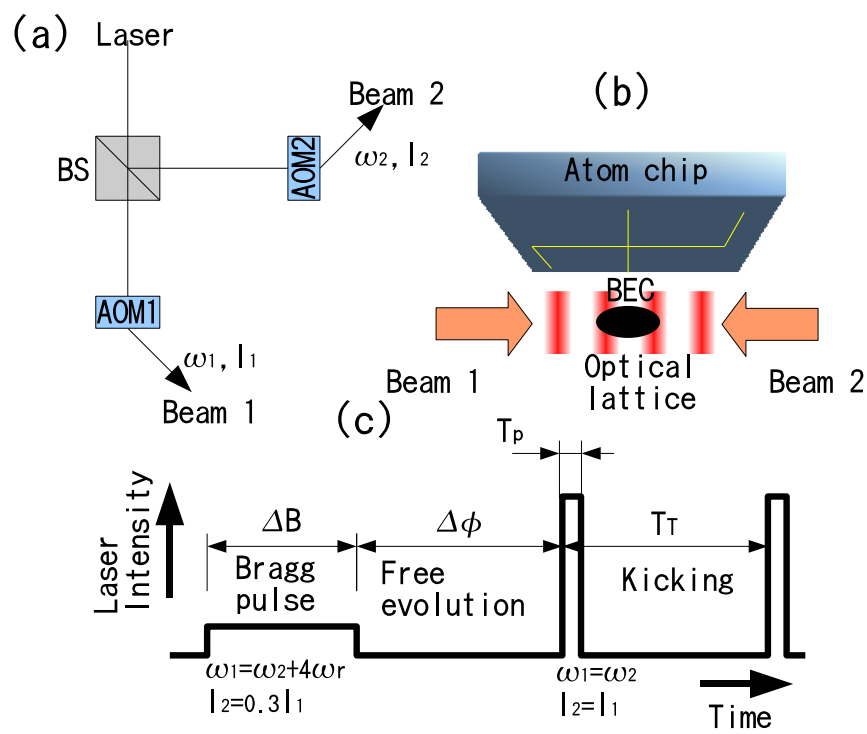

FIG. 1: Diagrams of the experimental setup and sequence. In (a), the laser configuration used to control Bragg diffraction and the kicking beam is shown. The beam is split by a $50 / 50$ beam splitter (BS) and the output light passes through separate acousto optic modulators (AOM) which control the beam intensities $I_{1,2}$ and frequencies $\omega_{1,2}$. The atom chip BEC setup is shown schematically in (b) along with the optical lattice created by the two intersecting beams. The three different phases of Bragg diffraction, phase evolution and kicking are shown in (c) and are explained further in the text.

or the period of the kicks, (which is set to the Talbot time $T_{T}$ corresponding to quantum resonance [13]). Rather, the observed directed diffusion is a property of the initial atomic wavefunction (which we prepare before kicking) in the presence of a resonantly pulsed optical lattice. The experiment cannot be performed with thermal atoms, as it requires an initial atomic momentum spread much less than a photon recoil in order to avoid dephasing effects. Our work presents analytical, simulation and experimental results for a specific realisation of a ratchet at $\mathrm{QR}$ similar to that proposed in [11]. We also offer a clear physical interpretation in terms of matter-wave interference.

As shown in Fig. 1] our experiment is comprised of a 

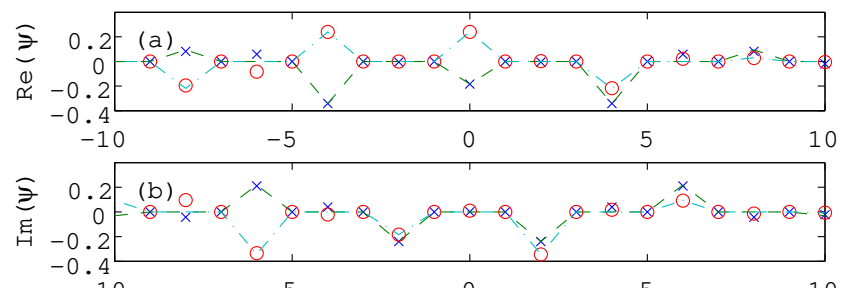

$\begin{array}{rrrrr}-0 & -10 & -5 & 0 & 5\end{array}$
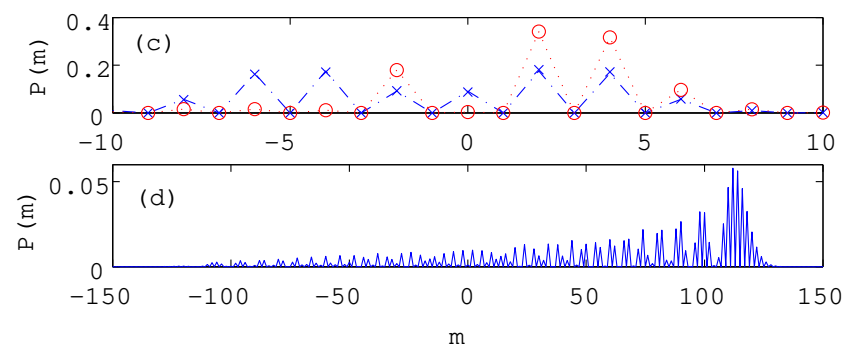

FIG. 2: Wavefunctions and momentum probability distributions for kicked atoms with $K=0.6$. The momentum $m$ is in units of $2 \hbar k_{l}$. In (a) and (b) respectively the real and imaginary parts of atomic wavefunctions after 5 kicks for $\phi=\pi$ are shown. The wavefunction evolving from an initial $\left|0 \hbar k_{l}\right\rangle$ state is shown with x's (simulations) and a dot-dash line (theory of Eq. 3), whilst that which evolved from an initial $\left|-2 \hbar k_{l}\right\rangle$ state is shown with o's (simulations) and a dotted line (theory). The lines are merely to guide the eye, and the theoretical wavefunction is only non-zero at multiples of $m=2 \hbar k_{l}$. In (c) asymmetry is seen to arise in the final momentum probability distribution corresponding to $\phi=\pi$ (dashed line - theory, squares - simulations) whilst for $\phi=\pi / 2$ (solid line - theory, triangles - simulations) there is symmetry about $m=-\hbar k_{l}$. In (d) the same system is shown after 100 kicks emphasising the extreme asymmetry of the momentum distribution.

BEC which is subjected to pulses from an optical standing wave. The experimental configuration has been explained elsewhere [14, 15] and thus we provide only a summary here. A BEC of $\sim 3 \times 10^{3}{ }^{87} \mathrm{Rb}$ atoms is realised and loaded onto an atom chip [15]. The atoms are trapped in the $5 S_{1 / 2}, F=2, m_{f}=2$ state by the magnetic field generated by the chip and sit $700 \mu \mathrm{m}$ below the chip surface. Typically, the axial trapping frequency for the BEC is $\omega_{z} \approx 2 \pi \times 17 \mathrm{~Hz}$ and the axial and radial Thomas-Fermi radii are $d_{z}=17 \mu \mathrm{m}$ and $d_{\rho}=3 \mu \mathrm{m}$ respectively. The BEC is prepared in an initial superposition state using a Bragg $\pi / 2$ pulse and then kicked using light from a diode laser. Fig. 1(a) shows the configuration used to control the intensity and frequency of the two beams used to create the Bragg and kicking pulses. A free running $100 \mathrm{~mW}$ diode laser, red detuned $4 \mathrm{GHz}$ (i.e. $\lambda=780.233 \mathrm{~nm}$ ) from the ${ }^{87} \mathrm{Rb} 5^{2} S_{1 / 2} \rightarrow 5^{2} P_{3 / 2}$ transition, enters a $50 / 50$ beam splitter and the output beams are passed through separate acousto-optic modulators (AOMs) to control their frequency and amplitude after which, they intersect with the BEC (Fig. 1(b)). The experimental sequence of laser pulses is shown in the diagram in Fig. 1(c).
For the Bragg pulse, the intensity of one beam is dropped to $3 \%$ of its maximum power using the amplitude modulation (AM) mode of one function generator whilst the frequency of the counterpropagating beam is increased by $4 \omega_{r} \approx 15 \mathrm{kHz}$ (where $\omega_{r}=2.37 \times 10^{4} \mathrm{~Hz}$ is the recoil frequency of ${ }^{87} \mathrm{Rb}$ ) relative to the other beam. After the Bragg pulse, a period $\Delta_{\phi}$ of free evolution is used to adjust the quantum phase of the $\left|-2 \hbar k_{l}\right\rangle$ state relative to $\left|0 \hbar k_{l}\right\rangle$, and the beam intensity and frequencies are made equal for kicking. The overall pulse envelope and timing were controlled by another pulse generator. The Bragg/kicking beams have an optical power of about $5 \mathrm{~mW}$. For a $\pi / 2$ pulse, a duration $\Delta_{B}$ of $60 \mu \mathrm{s}$ was used. For the kicking pulses, a width of $T_{p}=5 \mu \mathrm{s}$ was used with a pulse period $T$ equal to the Talbot time $T_{T}=\pi / 2 \omega_{r} \approx 66.3 \mu \mathrm{s}$ for ${ }^{87} \mathrm{Rb}$. Like other groups performing kicked BEC experiments [12], we have found that neither the energy due to atom-atom interactions nor the harmonic potential affect our results for the time scales used here, given the relatively much greater energy due to kicking of the atoms. We simulate the system by calculating the evolution of the initial wavefunction subject to the single atom Hamiltonian 2 (i.e. simulation of the Gross-Pitaevskii equation is not neccessary).

We now provide a theoretical treatment of our system. First we consider the preparation of the initial state by a Bragg $\pi / 2$ pulse. We will assume the the BEC starts in an initial 0 momentum eigenstate $\left|0 \hbar k_{l}\right\rangle$. This is not a bad approximation, since the atoms in the BEC have a thermal spread which is much less than $2 \hbar k_{l}$. The $\pi / 2$ pulse creates an equally weighted superposition state $\left|\psi_{B}\right\rangle=\frac{1}{\sqrt{2}}\left(\left|0 \hbar k_{l}\right\rangle-\mathrm{i}\left|2 \hbar k_{l}\right\rangle\right)$. After the Bragg pulse has been applied, a period $\Delta_{\phi}$ of free evolution is allowed. During this time, the $\left|-2 \hbar k_{l}\right\rangle$ state accumulates a phase $\phi=4 \omega_{r} \Delta_{\phi}$, where $\phi=2 \pi$ corresponds to $\Delta_{\phi}=T_{T}$. The initial state just before kicking starts is then

$$
\left|\psi_{i}\right\rangle=\frac{1}{\sqrt{2}}\left(\left|0 \hbar k_{l}\right\rangle-\mathrm{ie}^{i \phi}\left|-2 \hbar k_{l}\right\rangle\right)
$$

The dynamics due to sharp periodic momentum kicks applied to this state are governed by the Hamiltonian [16]

$$
\hat{H}=\frac{\hat{p}^{2}}{2}+K \cos \left(2 k_{l} \hat{x}\right) \sum_{t} \delta\left(t^{\prime}-t \tau\right),
$$

where $\hat{p}$ and $\hat{x}$ are the atomic momentum and position operators respectively, $K=\hbar V_{0} T_{p} / \hbar$ is the kicking strength for an optical potential of height $V_{0}, t^{\prime}$ is time, $t$ is the kick counter and $\tau=4 \pi T / T_{T}$ is the scaled kicking time. The associated Floquet operator for the case of QR $(\tau=4 \pi)$ is [17] $\hat{U}_{\text {q.r. }}(t)=\exp \left(-\mathrm{i} K t \cos \left(2 k_{l} \hat{x}\right)\right)$. Applied to $\left|\psi_{i}\right\rangle$, the output wavefunction $\psi_{o}$ and momentum distribution $P(m)$ are [18]

$$
\begin{aligned}
\psi_{o}(m)= & \frac{\mathrm{e}^{-i \frac{\pi}{2} m}}{\sqrt{2}}\left(J_{m}(K t)-\mathrm{e}^{i(\phi)} J_{m+1}(K t)\right), \\
P(m)= & \frac{1}{2}\left(J_{m}^{2}(K t)+J_{m+1}^{2}(K t)\right. \\
& \left.-2 \cos \phi J_{m}(K t) J_{m+1}(K t)\right) .
\end{aligned}
$$




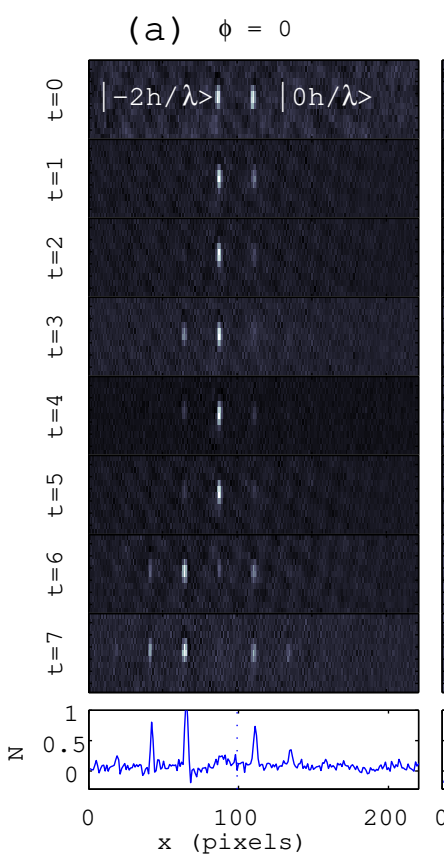

(b) $\phi=\pi / 2$
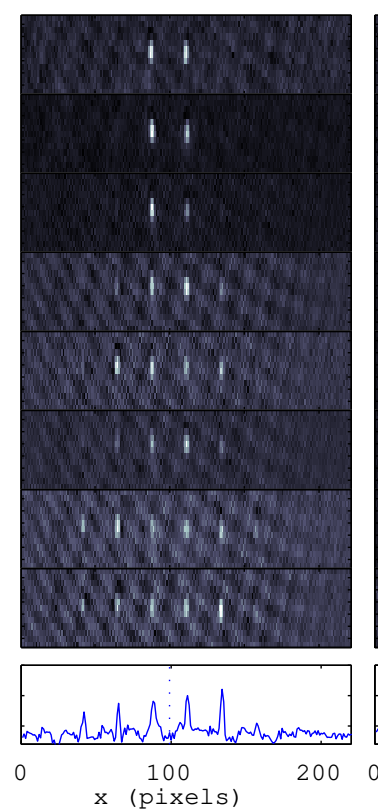

(C) $\phi=\pi$

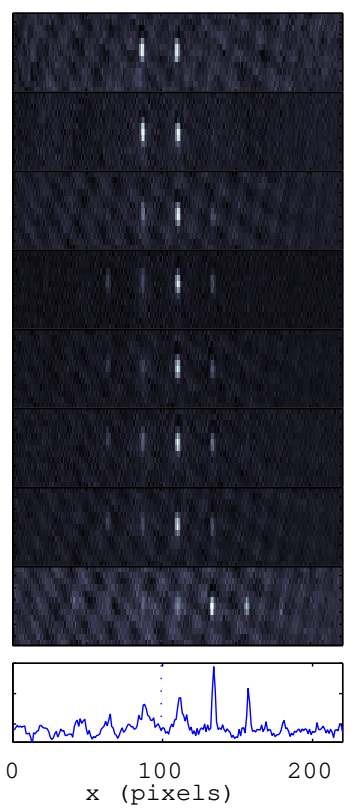

FIG. 3: Sequences of absorption images for the ratchet BEC experiment from $t=0$ to $t=7$ kicks for $(\mathrm{a}) \phi=0$, (b) $\phi=\pi / 2$ and (c) $\phi=\pi$. The $t=0$ case shows the initial distribution after the Bragg $\pi / 2$ pulse. In the top panel of (a), the 0 and $-2 \hbar k$ momentum states are shown. At the bottom of each column, a sum over the rows of the image for $t=7$ is shown giving the distribution of atom number $N$ with position. In these plots, the dashed line marks the position of the mean initial momentum $\hbar k$. The images clearly show the presence of a ratchet current which reverses direction when the phase $\phi$ crosses $\pi=\phi / 2$ (for which phase the current is seen to vanish).

Eq.s 3 and 4 have a particularly interesting property: for general phase $\phi$, the wavefunction and thus the momentum distribution grow asymmetrically with time. This property is seen in Figs. 2(a) and (b) which show the wave function after 5 kicks for $\phi=\pi$. The change in net momentum may be seen to be due to interference between the diffraction orders of the two initial wavefunctions which is mostly destructive below $m=-1$ but constructive above this initial mean momentum, leading to an asymmetric distribution of atoms (Fig. 2(c)). The dramatic nature of this induced asymmetry is demonstrated even more clearly in (Fig. 2(d)) which shows the theoretical probability distribution after 100 kicks. We note that the directed transport of atoms has been caused by the interference of diffracted matter waves, that is, the observed "ratchet" effect is entirely quantum (indeed, our experiment may be viewed as a type of atom interferometer [19]). Experimental confirmation is presented in Fig. 3 which shows absorption images of a kicked BEC after preparation into state $\psi_{i}$. The behaviour seen matches that predicted by Eq. 4. In particular, for $\phi=0$ the atomic momentum distribution increases in asymmetry towards negative momentum, whereas for $\phi=\pi$, the asymmetry is in the opposite direction. For $\phi=\pi / 2$ the distribution almost symmetrical (allowing for experimental fluctuations).

We may also find the momentum current $i(t)=(\mathrm{d} / \mathrm{d} t)\langle p(t)\rangle$ by calculating the first moment of the momentum distribution $\langle p\rangle=$ $\sum_{m} m P(m)=\frac{1}{2} \sum_{m}\left(m J_{m}^{2}(K t)+m J_{m+1}^{2}(K t)-\right.$ $\left.2 \cos (\phi) m J_{m}(K t) J_{m+1}(K t)\right)$. The first two terms give the momenta of the two superposed initial states e.g. 0 and -1 (in $2 \hbar k_{l}$ units) respectively. The term of interest is $\sum_{m} m J_{m}(K t) J_{m+1}(K t)$, which may be summed by applying the standard Bessel recursion formula and the Neuman sum rule 20] to give $K t / 2$. Thus

$$
i(t)=\frac{\mathrm{d}}{\mathrm{d} t}\langle p(t)\rangle=-\cos \phi \frac{K}{2} .
$$

Eq. [5 offers a useful way to summarise the data. The atomic momentum distribution was reconstructed from the absorption images shown in Fig. 3 and the mean momentum calculated. To check repeatability we took another set of data for the same parameters as those in Fig. 3. for $t>2$ (since very little diffusion occurs in the first two kicks). Average currents for the two data sets are shown in Fig. 4, along with error bars showing the difference between the measurements. The extraction of very small mean momenta $\left(\langle p\rangle \sim \hbar k_{l}\right)$ from the ditributions in Fig. 3 is hampered by experimental imperfections such as CCD noise and scattered light, and laser frequency drift, which may lead to ocassional changes in experimental parameters. This is the most likely cause of the large error bar seen in the case of $\phi=\pi$ when $t=6$. 


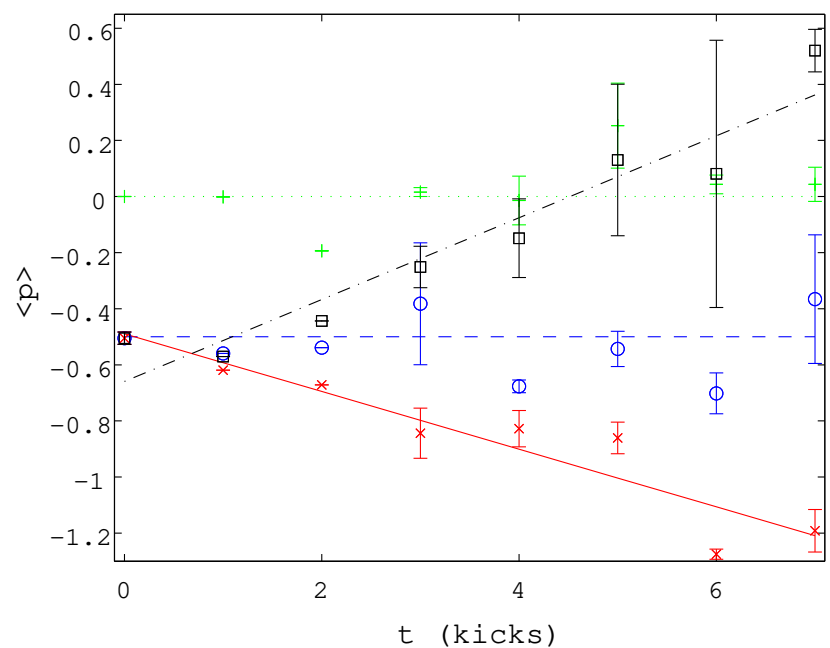

FIG. 4: The experimentally measured $\langle p\rangle$ (in units of $2 \hbar k_{l}$ ) is shown along with theoretical curves for various initial conditions. Experimental data is shown by $\mathrm{x}(\phi=0)$, ० $(\phi=\pi / 2)$, $\square(\phi=\pi)$ and + (no initial $\pi / 2$ pulse $)$. The solid and dashdot lines are fits to the data for $\phi=0$ and $\phi=\pi$ respectively. Dashed and dotted lines show $\langle p\rangle=-0.5$ and $\langle p\rangle=0$ respectively (note that these lines are not fits to the data).

Increasing the accuracy of the measurements would require a larger atom number and ideally a separate laser for Bragg diffraction and kicking. Nonetheless, Fig. 4 clearly demonstrates the momentum current effect and a current reversal for $\phi=0$ compared with $\phi=\pi$. The data shows a general linear trend as predicted by Eq. 5 . with fitted lines shown in both cases. For $\phi=\pi / 2$, although individual momentum distributions are not per- fectly symmetrical, the current is near 0 on average. The control case for an initial $\left|0 \hbar k_{l}\right\rangle$ distribution is also shown and seen to exhibit near 0 average momentum current. Note that the dotted and dashed lines are not fits to the data, since there are no free parameters in either of these cases. Theoretically, the momentum current should persist indefinitely. In an experimental setting, however, imperfections such as the finite pulse width and any small difference between the pulse period and the Talbot time will reduce the ratchet current. Due to a low signal to noise ratio at higher kick numbers in the current experiment, it was not possible to probe these effects with our current setup. We note that the effects seen here require a well defined quantum phase between the initial states in superposition. Therefore, the experiment must be performed using a BEC as a thermal cloud typically has a large spread of initial momenta (and therefore quantum phase after free evolution), destroying the directed diffusion effect. It may be possible to exploit any sensitivity of the ratchet current to pulse-timing and phase variations to make accurate interferometry measurements.

In summary, we have demonstrated a novel quantum "ratchet" effect, in which directed momentum transport occurs in a system subject to a pulsed potential with no net bias. The effect has no classical analogue, unlike previous such systems studied experimentally. The direction of the ratchet current varied with the initial quantum phase as predicted, showing complete reversal for $\phi=0$ compared with $\phi=\pi$. This realisation of directed momentum transport suggests new possible mechanisms for directed motion on any scale where quantum interference effects are non-negligible and resonant transport exists. M.S. would like to thank Scott Parkins and Andrew Daley for discussions regarding this work.
[1] R.P.Feynman, The Feynman Lectures on Physics (Addison-Wesley, Reading, MA,1963), Vol. 1, Chap 46.

[2] R.D. Astumian and P. Hänggi, Phy. Today 55(11), 33 (2002).

[3] P. Riemann, Phys. Rep. 361, 57 (2002)

[4] P. Riemann, M. Grifoni and P. Hänggi, Phys. Rev. Lett. 79, 10 (1997).

[5] M. Schiavoni et al., Phys. Rev. Lett 90, 094101 (2003); G.C. Carlo, et al., ibid., 94, 164101 (2005); S.Flach, O. Yevtushenko and Y. Zolotaryuk, ibid., 84, 2358 (2000).

[6] H. Schanz et al., Phys. Rev. Lett. 87, 070601 (2001); J. Gong and P. Brumer, Phys. Rev. Lett. 97, 240602 (2006).

[7] C. Mennerat-Robilliard, et al., Phys. Rev. Lett. 82, 851 (1999) T.S. Monteiro et al., Phys. Rev. Lett. 89, 194102 (2002);

[8] G.C. Carlo, et al., Phys. Rev. A 74, 033617 (2006); D. Poletti, et al., cond-mat/0609535 (unpublished).

[9] P.H. Jones, et al., Phys. Rev. Lett. 98, 073002 (2007).

[10] F. L. Moore, et al., Phys. Rev. Lett. 75, 4598 (1995).

[11] E. Lundh and M. Wallin, Phys. Rev. Lett 94, 110603
(2005); D. Poletti, G.C. Carlo and B. Li, Phys. Rev. E 75, 011102 (2007); S. Denisov, L. Morales-Molina and S. Flach, cond-mat/0607558 (unpublished).

[12] GJ Duffy, et al., Phys. Rev. A 70, 041602(R) (2004) ;S. Wimberger, et al., Phys. Rev. Lett. 94, 130404 (2005);G. Behinaein, et al., Phys. Rev. Lett. 97, 244101 (2006).

[13] C. Ryu, et al., Phys. Rev. Lett. 96, 160403 (2006).

[14] M. Horikoshi and K. Nakagawa, Phys. Rev A 74, 031602(R) (2006).

[15] M. Horikoshi and K. Nakagawa, Appl. Phys. B 82, 363 (2006).

[16] R. Graham, M. Schlautmann and P. Zoller, Phys. Rev. A 45, 19(R) (1992).

[17] F.M. Izrailev and D.L. Shepelyanskii, Doklady Akademii Nauk SSSR 256, 586 (1981).

[18] D. Cohen, Phys. Rev. A 44, 2292 (1991).

[19] M.R. Andrews, et al., Science 275, 637 (1997).

[20] G.N. Watson, A treatise on the theory of Bessel functions, Cambridge Univ. Press (1996). 